\title{
Inhibitory Effects of S-carboxymethylcystein on Goblet Cell Proliferation in Cultured Epithelium
}

\author{
Satoshi Yamaguchi ${ }^{1}$, Shoji Matsune ${ }^{1}$, Kuwon Sekine ${ }^{1}$, Nozomu Wakayama ${ }^{1}$, \\ Yasuhiko Kitayama ${ }^{2}$, Yuji Nakamura ${ }^{2}$ and Kimihiro Okubo ${ }^{3}$ \\ ${ }^{1}$ Department of Otorhinolaryngology, Nippon Medical School Musashi Kosugi Hospital, Kanagawa, Japan \\ ${ }^{2}$ Department of Pathology, Nippon Medical School Musashi Kosugi Hospital, Kanagawa, Japan \\ ${ }^{3}$ Department of Otolaryngology, Nippon Medical School Hospital, Tokyo, Japan
}

\begin{abstract}
Background: The influence of S-carboxymethylcystein (S-CMC) on the proliferation ability of goblet cells in nasal polyp epithelium in response to inflammatory stimulation was examined.

Methods: The subjects were patients with chronic paranasal sinusitis. An epithelial cell culture system was established using nasal polyp mucosa excised during endoscopic paranasal sinus surgery. The samples were divided into 4 groups (group a: control group, group b: $10 \mathrm{ng} / \mathrm{mL}$ tumor necrosis factor- $\alpha$ (TNF- $\alpha$ ) treatment group, group c: $10^{-7} \mathrm{M} \mathrm{S}-\mathrm{CMC}$ and $10 \mathrm{ng} / \mathrm{mL}$ TNF- $\alpha$ treatment group, group $\mathrm{d}: 10^{-5}$ M S-CMC and $10 \mathrm{ng} / \mathrm{mL}$ TNF- $\alpha$ treatment group). The total number of epithelial cells and number of goblet cells were measured under a microscope, and the ratio of goblet cells to the total number of epithelial cells was calculated.

Results: In group b, $10 \mathrm{ng} / \mathrm{mL}$ of TNF- $\alpha$ significantly increased the number of goblet cells compared with group a, suggesting involvement of TNF- $\alpha$ in goblet cell proliferation. In addition, the number of goblet cells significantly decreased in group d compared with that in group $\mathrm{b}$, and it also decreased in group c compared with that in group b, although the difference was not significant, and the decrease was smaller than that in group $d$, suggesting that S-CMC inhibited goblet cell proliferation in a concentration-dependent manner.

Conclusion: TNF- $\alpha$ promoted goblet cell proliferation in nasal polyps, suggesting its influence on nasal polyp formation. As S-CMC inhibited inflammatory stimulation-induced goblet cell proliferation in nasal polyp epithelium, it may be useful for the treatment of sinusitis.
\end{abstract}

(J Nippon Med Sch 2018; 85: 215-220)

Key words: S-carboxymethylcystein, TNF- $\alpha$, chronic sinusitis, goblet cell, nasal polyp

\section{Introduction}

Several factors have been reported to be involved in the transition of paranasal sinusitis to a chronic state such as physical ventilatory and excretory disorders of the paranasal sinus due to infection, allergy, and nasal polyp formation in the paranasal sinus mucosa, as well as functional disorders of the mucociliary system ${ }^{1}$. Goblet cells in nasal polyp epithelium secrete mucus and are considered to influence mucociliary transport through mucin secretion in chronic sinusitis ${ }^{2}$. In addition, a potent ability to proliferate has been demonstrated by goblet cells in nasal polyp epithelium, suggesting their involvement in nasal polyp enlargement ${ }^{3}$. An inflammatory cytokine, tumor necrosis factor- $\alpha$ (TNF- $\alpha$ ), is detected in nasal discharge and the paranasal sinus mucosa in chronic sinusitis, indicating its involvement in the pathology of chronic sinusitis ${ }^{45}$; however, to our knowledge, its influence on goblet cell proliferation has not been reported.

In Japan, chronic sinusitis is conventionally treated with S-carboxymethylcystein (S-CMC) combined with macrolide therapy as conservative treatment. The pharmacological effects of S-CMC have been reported to be a

Correspondence to Satoshi Yamaguchi, Nippon Medical School Musashi Kosugi Hospital, 1-396 Kosugi-cho, Nakahara-ku,

Kawasaki, Kanagawa 211-8533, Japan

E-mail: s6095@nms.ac.jp

Journal Website (http://www2.nms.ac.jp/jnms/) 
Table 1 Subjects

\begin{tabular}{ccccc}
\hline Case & Age & Sex & Chief complaint & JESREC Score \\
\hline 1 & 46 & M & Nasal obstruction & 8 \\
2 & 74 & M & Postnasal drip & 5 \\
3 & 23 & M & Postnasal drip & 9 \\
4 & 66 & M & Nasal obstruction & 10 \\
\hline
\end{tabular}

All the subjects were male, aged 23-74 years. Their main complaints were nasal congestion and postnasal drip (2 cases each). JESREC scores were 5-10.

※JESREC Score: $\geq 11=$ eosinophilic sinusitis

(Tokunaga T. et al: Novel scoring system and algorithm for classifying chronic rhinosinusitis: the JESREC study. Allergy 2015: 70: 995-1003)

normalization of the mucus properties and improvement in mucociliary transport function ${ }^{6}$, but the effects on goblet cell proliferation have not been clarified.

In this study, using a culture system of nasal polyp epithelial cells collected from patients with surgically treated chronic sinusitis, the influence of TNF- $\alpha$ on goblet cell proliferation and the effects of S-CMC were examined.

\section{Materials and Methods}

\section{Subjects}

Four patients with chronic sinusitis underwent endoscopic paranasal sinus surgery between April 2012 and March 2013 at the Department of Otolaryngology, Nippon Medical School Musashi Kosugi Hospital. Nasal polyp epithelium excised during surgery was used. Patients with concomitant allergic rhinitis and bronchial asthma were excluded. All the patients were male, aged 23-74 years. Their chief complaints were nasal obstruction and postnasal drip (2 cases each). None of the patients was diagnosed with eosinophilic chronic rhinosinusitis (ECRS), with JESREC scores ${ }^{7}$ of 5-10 (Table 1).

\section{Methods}

(1) Collection and culture of nasal polyp epithelial cells Using nasal polyp mucosa excised from 4 patients with surgically treated chronic sinusitis, the epithelial cell culture system was prepared as follows: The collected nasal polyp was washed with phosphate buffer at $\mathrm{pH}$ 7.0, placed in $0.1 \%$ protease (Nacalai Tesque, Japan)containing transfer medium [100 mL of DMEM medium (Nacalai Tesque, Japan) $+100 \mathrm{~mL}$ of F12 medium (GIBCO, New York) + $2 \mathrm{~mL}$ of penicillin-streptomycin mixed solution (Nacalai Tesque, Japan)], and kept standing in a $37^{\circ} \mathrm{C}, 5 \% \mathrm{CO}_{2}$ incubator for $1.5 \mathrm{~h}$. The sample was taken out of the incubator and epithelial cells were detached by pipetting in the dish. Only the supernatant was collected and centrifuged at $980 \mathrm{rpm}$ for $3 \mathrm{~min}$. Only the supernatant was collected, washed with wash medium [100 mL of DMEM (Nacalai Tesque, Japan) + 100 $\mathrm{mL}$ of F12 medium (GIBCO, New York) $+2 \mathrm{~mL}$ of penicillin-streptomycin mixed solution (Nacalai Tesque, Japan) $+2 \mathrm{~mL}$ of fetal bovine serum (GIBCO, New York)], and centrifuged again at $980 \mathrm{rpm}$ for $3 \mathrm{~min}$. The supernatant was collected and kept standing in a $37^{\circ} \mathrm{C}$, $5 \% \mathrm{CO}_{2}$ incubator for $1 \mathrm{~h}$. As fibroblasts adhered to the dish bottom, the supernatant containing floating epithelial cells was collected. The cells were washed with subculture medium [500 mL of BEBM (LONZA, Switzerland) +1 BEGM Single Quots kit (LONZA, Switzerland) + 300 $\mathrm{mg}$ of bovine serum albumin (Sigma, Missouri) $+12.5 \mu \mathrm{g}$ of epidermal growth factor] and centrifuged at $980 \mathrm{rpm}$ for $3 \mathrm{~min}$. The supernatant was collected, combined with subculture medium, mixed, and cultured in a $37^{\circ} \mathrm{C}, 5 \%$ $\mathrm{CO}_{2}$ incubator. Subculture medium was replaced every 2 days, and after confirming an epithelial cell monolayer was being cultured on the plate, the experiment was performed following the protocol below:

(2) Protocol for drug administration experiment (Fig. 1)

1) Cultured nasal polyp epithelium was divided into 4 groups (group a: control group, group b: TNF- $\alpha$ treatment group, group c: S-CMC $10^{-7} \mathrm{M}$ and TNF- $\alpha$ treatment group, group d: S-CMC $10^{-5} \mathrm{M}$ and TNF- $\alpha$ treatment group). After 4-day culture, groups a and b were left untreated, and S-CMC was administered to the nasal polyp epithelium at S-CMC $10^{-7} \mathrm{M}$ in group $\mathrm{c}$ and $\mathrm{S}$ $\mathrm{CMC} 10^{-5} \mathrm{M}$ in group $\mathrm{d}$.

2) After 48 hours, $10 \mathrm{ng} / \mathrm{mL}$ of TNF- $\alpha$ was administered to groups b, c, and $d$. The dose of TNF- $\alpha$ was decided based on the level previously measured in fluid retained in the paranasal sinus (unpublished data). 


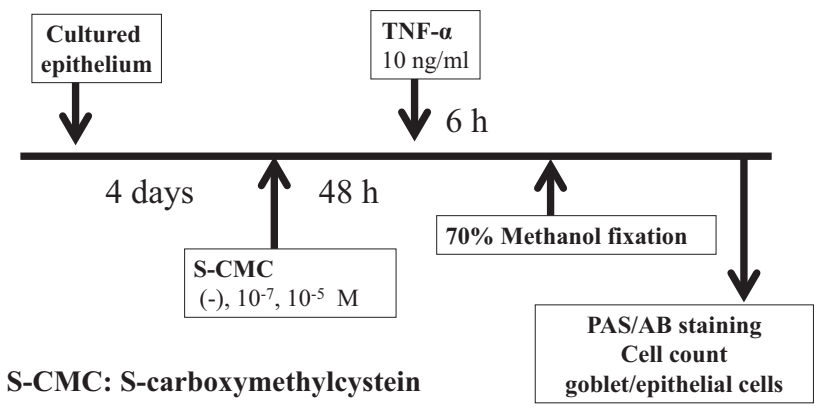

Fig. 1 Study methods

The nasal polyps collected were divided into 4 groups (group a: control group, group b: tumor necrosis factor- $\alpha$ (TNF- $\alpha$ ) treatment group, group c: S-carboxymethylcystein (S-CMC) $10^{-7} \mathrm{M}$ and TNF- $\alpha$ treatment group, group d: SCMC $10^{-5} \mathrm{M}$ and TNF- $\alpha$ treatment group). Only nasal polyp epithelial cells were extracted and cultured for 4 days. Groups a and b were not treated, while groups c and d were treated with S-CMC $10^{-7} \mathrm{M}$ and $10^{-5} \mathrm{M}$, respectively. After 48 hours, $10 \mathrm{ng} / \mathrm{mL}$ TNF- $\alpha$ was added to groups b, c, and d. After 6 hours, the total number of epithelial cells and number of goblet cells were measured, and the ratio of the number of goblet cells to that of epithelial cells was compared among the 4 groups.

3) The cultured nasal polyp epithelial cells were fixed with $70 \%$ methanol 6 hours after TNF- $\alpha$ administration and subjected to Alcian Blue-PAS staining. In addition, the dynamics of the cultured cells were video-recorded under a phase-contrast microscope. The total number of epithelial cells and number of goblet cells were counted, and the ratio of goblet cells in the total number of epithelial cells was calculated. Cells were counted by a pathologist blinded to the study background.

(3) Statistical analysis

Student's t-tests were used for statistical analysis.

As this was a study using surgically collected human samples, it was performed after approval by the Ethics Committee of Nippon Medical School Musashi Kosugi Hospital (Permission number: 219-24-19) and obtaining consent from the patients.

\section{Results}

Establishment of an epithelial cell culture system with ciliary movement was confirmed under a phase-contrast microscope. Microscopic images are presented in Fig. 2. The number of epithelial cells (N-EC), the number of goblet cells (N-GC), and the ratios of the number of goblet cells to the total number of epithelial cells (number of goblet cells/number of epithelial cells: N-GC/N-EC) were 680.5, 4.70, and 7.12 in group a, 604.9, 5.90, and 10.24 in group b, 876.8, 7.50, and 8.66 in group $c$, and
$476.8,3.30$, and 6.81 in group d. The N-GC/N-EC ratios were compared among the four groups (Fig. 3). The ratio was significantly higher in group $b$ than a $(p<0.05)$, suggesting involvement of TNF- $\alpha$ in goblet cell proliferation. The mean ratio decreased in the order of group b, c, and $\mathrm{d}$, and it was significantly decreased in group $\mathrm{d}$ compared with that in group $b(p<0.05)$, suggesting that $S$ CMC inhibited goblet cell proliferation in a concentration-dependent manner.

\section{Discussion}

In Japan, macrolides are the first-choice for drug therapy for chronic sinusitis. In general, expectorants, such as $\mathrm{S}-$ $\mathrm{CMC}$, steroids, and anti-allergic drugs, are combined with macrolide therapy. Several factors have been reported to be involved in the transition of paranasal sinusitis to a chronic state: Bacterial and viral infections, and allergic reactions inducing inflammatory reactions in the paranasal sinus mucosa, reduction in the fluid retained in the paranasal sinus due to mucociliary transport by the nasal mucosa affected by local anatomical factors and secretions, and activation of inflammatory agents such as bacteria-derived substances, immune complexes, and cytokines ${ }^{2,8-10}$. Nasal polyps are formed in many cases, and possible causes are edema induced by promoted vascular permeability ${ }^{11}$, epithelial rupture ${ }^{12}$, and action of matrix metalloproteinase, but no cause has been clarified. Nasal polyp mucosa consists of epithelial tissue and interstitium. Epithelial tissue is pseudostratified epithelium containing goblet cells, similar to other respiratory epithelium, and the number of goblet cells varies depending on the nasal polyp development $\operatorname{site}^{13}$. In the interstitium, proliferation of gland tissue, vascular endothelial cells, fibroblasts, and collagen are observed in addition to infiltrating inflammatory cells. The association of nasal polyp proliferation with several cytokines and growth factors has been reported, and production of different cytokines and chemokines by fibroblasts isolated from nasal polyps has been demonstrated, suggesting their involvement in nasal polyp proliferation ${ }^{14}$. Goblet cells, abundantly present in nasal polyp epithelial tissue, are mucus-secreting cells, and they have been reported to be involved in mucin secretion in chronic sinusitis ${ }^{2}$. Goblet cells in nasal polyp epithelium have high proliferative ability, and proliferation of secretory goblet cells, in addition to epithelial cells, may be involved in epithelial hyperplasia during inflammation ${ }^{3}$. On the other hand, abundant TNF- $\alpha$, an inflammatory cytokine, in nasal discharge and paranasal sinus mucosa in chronic sinusitis 


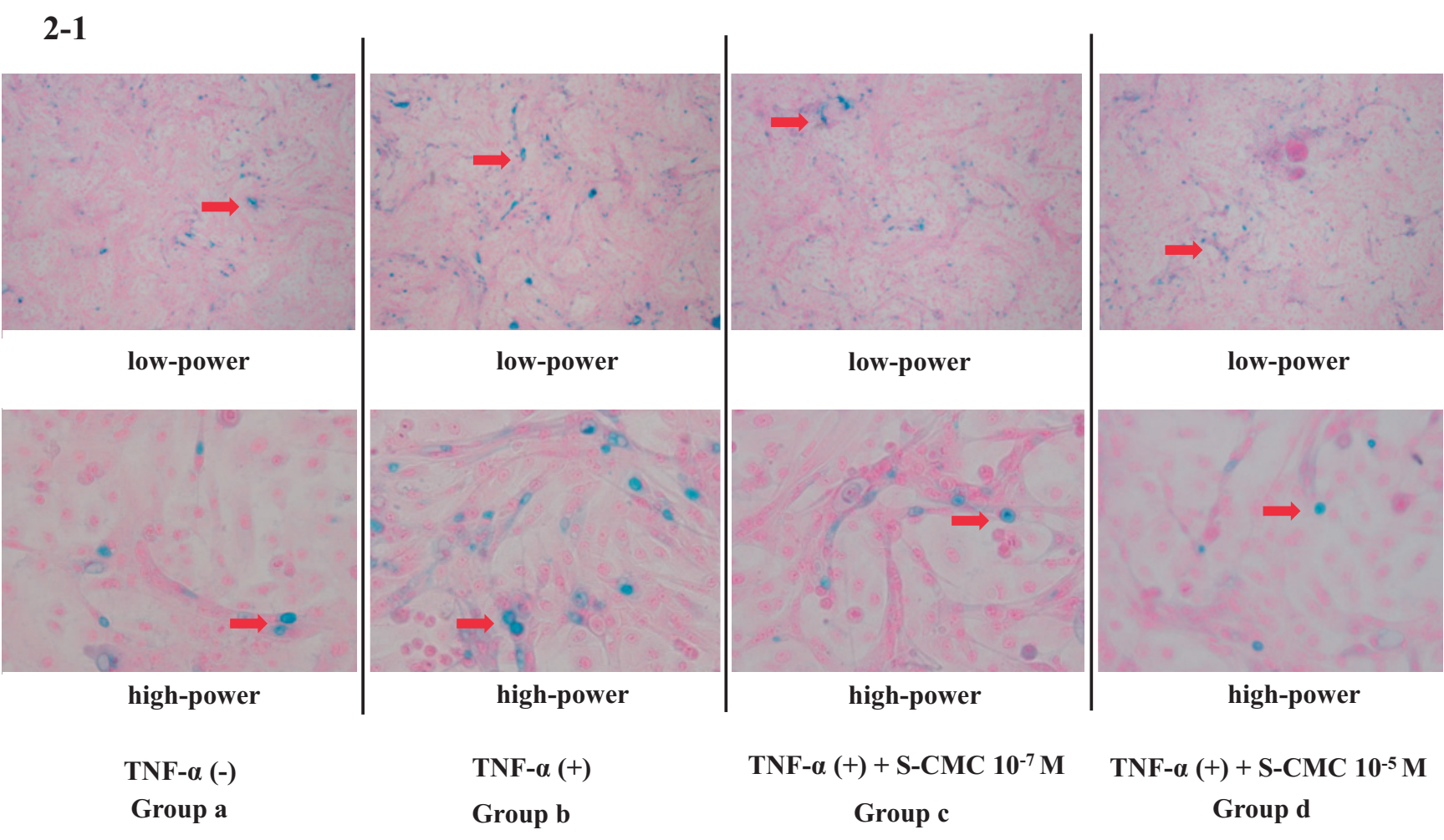

\section{2-2}

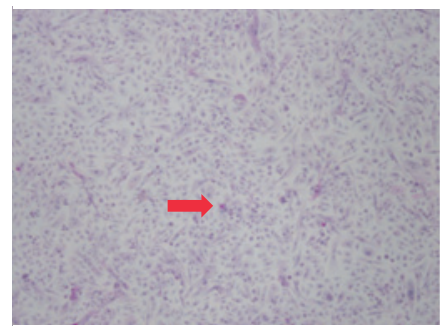

low-power

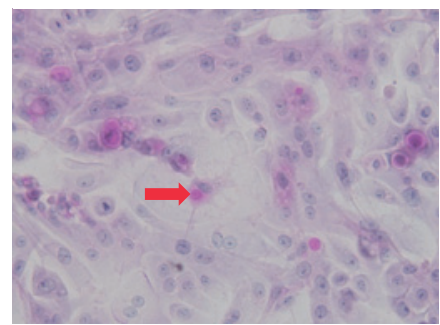

high-power

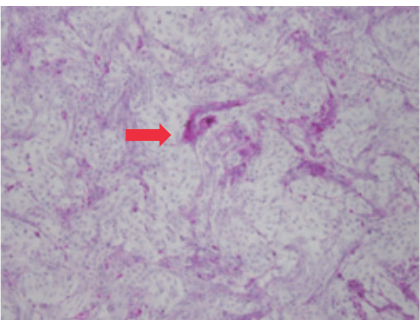

low-power

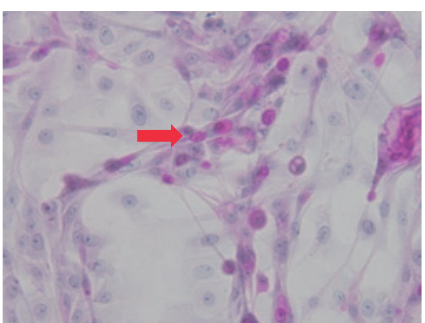

high-power

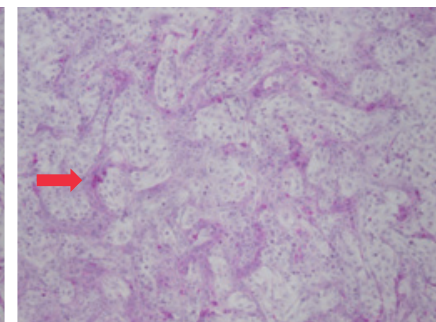

low-power

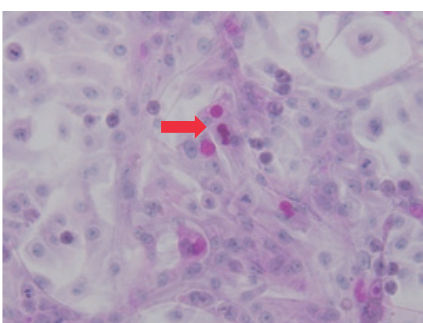

high-power

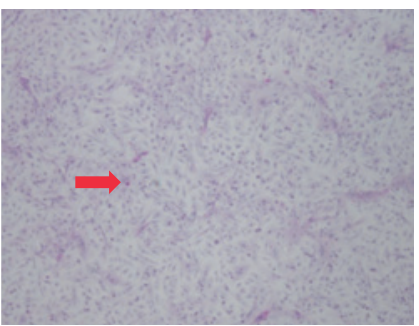

low-power

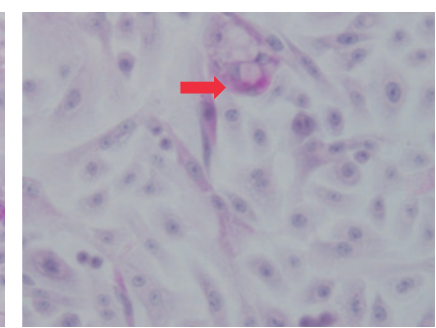

high-power

\section{Goblet cell}

TNF- $\alpha(+)+$ S-CMC $10^{-7} \mathrm{M}$

Group c
TNF- $\alpha(+)+$ S-CMC $10^{-5}$ M

Group d

Group a

Group b

Fig. 2 Microscopic images (Fig. 2-1: Alcian blue staining Fig. 2-2: PAS staining)

Fig. 2-1 shows a microscopic image made of Alcian blue staining, and Fig. 2-2 shows that of PAS staining. The microscope images show the low-powered field on the upper level, and the high-powered field on the lower level. Acidic mucus produced from goblet cells was increased in group b, and reduced in group c and group $\mathrm{d}$.

has been demonstrated, suggesting its involvement in the pathology of chronic sinusitis ${ }^{4,5}$. TNF- $\alpha$ is generated by stimuli, such as viral and bacterial infections, to promote the release of granulocyte / macrophage colony- 


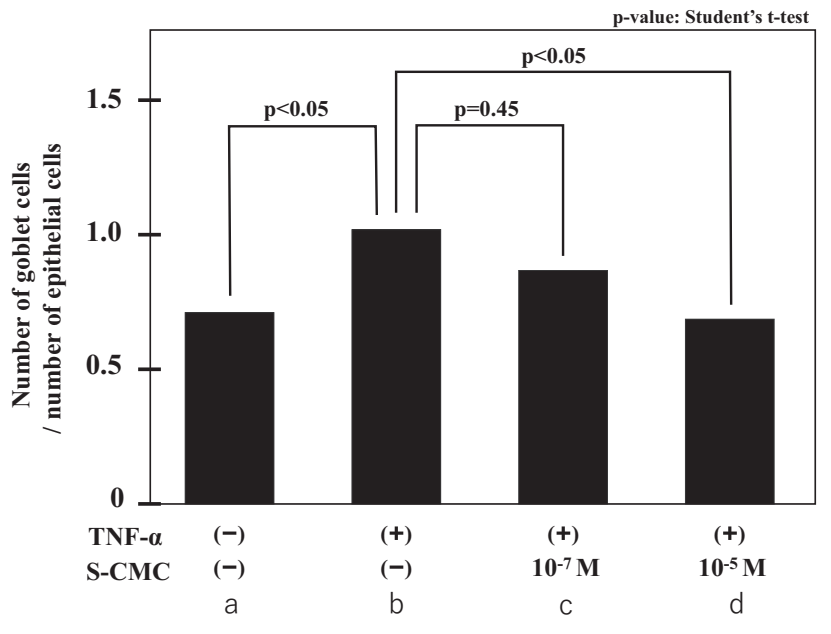

Fig. 3 Results

In the non-S-carboxymethylcystein (S-CMC) treatment groups (groups a and b), the ratio of goblet cells to the total number of epithelial cells was significantly increased by tumor necrosis factor- $\alpha($ TNF- $\alpha)(p<0.05)$. In the TNF- $\alpha$ treatment groups (groups b, c, and d), the mean was slightly lower in the S-CMC $10^{-7} \mathrm{M}$ treatment group (group c) than in the non-S-CMC treatment group (group $b)(p=0.45)$, and significantly lower in the S-CMC $10^{-5} \mathrm{M}$ treatment 10 group (group $\mathrm{d})(\mathrm{p}<0.05)$. The ratio of goblet cells to epithelial cells was lower in the S-CMC $10^{-5} \mathrm{M}$ group than in the $10^{-7} \mathrm{M}$ treatment group (group c), suggesting that S$\mathrm{CMC}$ inhibits goblet cell proliferation in a concentrationdependent manner.

stimulating factor (GM-CSF) from the nasal mucosal epithelium and fibroblasts. GM-CSF promotes eosinophil activation $^{15}$ and enhances GM-CSF mRNA expression in nasal polyps ${ }^{16}$. The number of eosinophils is correlated with the number of GM-CSF-producing cells in nasal polyps ${ }^{17}$. Therefore, GM-CSF produced upon TNF- $\alpha$ stimulation may play an important role in eosinophil accumulation in nasal polyps. TNF- $\alpha$ also induces the expression of adhesion molecules (intercellular adhesion molecule- 1 and vascular cell adhesion molecule-1) in vascular endothelial cells and promotes eosinophil migration ${ }^{18}$. Thus, goblet cells in the nasal polyp epithelium may be involved in eosinophil infiltration, and TNF- $\alpha$ may promote eosinophil infiltration in nasal polyps and induce goblet cell proliferation. However, no report has been published on these hypotheses. In the present study, TNF- $\alpha$ administration to cultured nasal polyp epithelial cells significantly increased the number of goblet cells, suggesting that TNF- $\alpha$ is involved in goblet cell proliferation by acting on undifferentiated cells among epithelial cells.

Concerning drug therapy for chronic sinusitis, in addition to infection control in the paranasal sinus, improvement in the self-cleaning action of the paranasal sinus, such as mucociliary transport and shrinkage of nasal polyps, is important. S-CMC, which is frequently used in combination with macrolide therapy, improves mucociliary transport ${ }^{19}$, and improvement of subjective nasal discharge has also been reported, demonstrating its usefulness for treatment ${ }^{6}$. Inhibitory effects on goblet cells among nasal polyp epithelial cells were observed, but goblet cell inhibitory effects by S-CMC in normal nasal and paranasal sinus mucosae were not observed ${ }^{19}$. Carbocysteine does not merely decrease goblet cells, but decreases and normalizes pathologically hyperplastic goblet cells. Goblet cells significantly proliferated in chronic sinusitis with marked eosinophil infiltration, and mucus is caused by excessive secretion from goblet cells ${ }^{20}$. Carbocysteine suppresses mucin secretion in nasal discharge ${ }^{21}$. Thus, carbocysteine reduces excessive mucin secretion from goblet cells through normalization of goblet cell proliferation, thereby normalizing mucociliary transport.

\section{Conclusion}

Changes in the number of goblet cells in cultured nasal polyp epithelium induced by TNF- $\alpha$ and S-CMC were examined. TNF- $\alpha$ significantly increased the number of goblet cells, suggesting that TNF- $\alpha$ is involved in goblet cell proliferation and influences nasal polyp formation. SCMC decreased the number of goblet cells in cultured nasal polyp epithelium in a concentration-dependent manner. Thus, S-CMC may normalize goblet cell proliferation in the nasal polyp epithelium and may also be involved in the normalization of mucociliary transport.

Conflict of Interest: The authors have no conflicts of interest directly relevant to the content of this article. And the authors have not received any funding in describing this article.

\section{References}

1. Ikeda K: Pathophysiology and Treatment Strategy of Chronic Rhinosinusitis with Nasal Polyps. Practica OtoRhino-Laryngologica 2013; 106: 573-580.

2. Majima Y: Pathogenesis of mucus hypersecretion and its treatments in chronic sinusitis. Japanese Journal of Rhinology 2007; 46: 50-56.

3. Iida M: Immunohistochemical Analysis of Proliferating Cell Nuclear Antigen Expression in Human Nasal Polyp. Oto-rhino-laryngology Tokyo. 1998; 41: 15-20.

4. Furukido K: Treatment of Sinusitis with the YAMIK Sinus Catheter. Oto-rhino-laryngology Tokyo. 2003; 46: 34-40.

5. Ueda T: Cytokine of Paranasal Sinus Effusion Taken by the YAMIK Sinus Catheter. Oto-rhino-laryngology Tokyo. 2001; 44: 88-96.

6. Baba S: Clinical evaluation of Carbocisteine in the treatment of chronic paranasal sinusitis A double blind trial comparing with L-cysteine ethylester hydrochloride. 
Otologia Fukuoka 1988; 33-47.

7. Tokunaga T, Sakashita M, Haruna T, Asaka D, Takeno S, Ikeda H, Nakayama T, Seki N, Ito S, Murata J, Sakuma Y, Yoshida N, Terada T, Morikura I, Sakaida H, Kondo K, Teraguchi K, Okano M, Otori N, Yoshikawa M, Hirakawa K, Haruna S, Himi T, Ikeda K, Ishitoya J, Iino Y, Kawata R, Kawauchi H, Kobayashi M, Yamasoba T, Miwa T, Urashima M, Tamari M, Noguchi E, Ninomiya T, Imoto Y, Morikawa T, Tomita K, Takabayashi T, Fujieda S: Novel scoring system and algorithm for classifying chronic rhinosinusitis: the JESREC Study. Allergy 2015; 70: 995-1003.

8. Tos M, Mogensen C: Mucus production in chronic maxillary sinusitis: a quantitative histopathological study. Acta oto-laryngologica 1984; 97: 151-159.

9. Atsuta S, Majima Y: Nasal mucociliary clearance of chronic sinusitis in relation to rheological properties of nasal mucus. Annals of Otology, Rhinology \& Laryngology 1998; 107: 47-51.

10. Majima Y: Clinical implications of the immunomodulatory effects of macrolides on sinusitis. The American Journal of Medicine Supplements 2004; 117: 20-25.

11. Kakoi H: A histological study of formation and growth of nasal polyps. Acta oto-laryngologica 1987; 103: 137-144.

12. Tos M: Mucous glands in nasal polyps. Archives of otolaryngology (1960) 1977; 103: 407-413.

13. Tos M: Goblet cell density in nasal polyps. Annals of otology, rhinology \& laryngology 1990; 99: 310-315.

14. Suzaki H: Influence of roxithromycin on inflammatory cytokine production from nasal polyp fibroblasts in vitro. Acta oto-laryngologica 2003; 123: 637-642.

15. Gauldie J, Cox G, Jordana M, Ohno I, Kirpalani H: Growth and Colony-Stimulating Factors Mediate Eosinophil Fibroblast Interactions in Chronic Airway Inflammation. Annals of the New York Academy of Sciences 1994;
725: 83-90.

16. Ohno I, Lea R, Finotto S, Marshall J, Denburg J, Dolovich J, Gauldie J, Jordana M: Granulocyte/macrophage colonystimulating factor (GM-CSF) gene expression by eosinophils in nasal polyposis. Am J Respir Cell Mol Biol 1991; 5: 505-510.

17. Hamilos DL, Leung DY, Wood R, Meyers A, Stephens JK, Barkans J, Meng Q, Cunningham L, Bean DK, Kay AB, Hamid Q: Chronic hyperplastic sinusitis: association of tissue eosinophilia with mRNA expression of granulocyte-macrophage colony-stimulating factor and interleukin-3. Journal of allergy and clinical immunology 1993; 92: 39-48.

18. Haruna S, Otori N, Yanagi K: Epithelial cell injury in chronic sinusitis with nasal polyp. Japanese Journal of Rhinology 2000; 39: 352-358.

19. Majima Y: Effect of S-carboxymethylcysteine on Nasal Mucociliary Clearance and Viscoelasticity of Nasal Mucus. The Oto-rhino-and laryngological clinic Practica otologica Kyoto 1987; 80: 1313-1319.

20. Fukami M, Yanagi K: Classification of Chronic Sinusitis on the Basis of Eosinophil Infiltration as an Additional Factor. Japanese Journal of Rhinology 1999; 38: 441-450.

21. Yasuo M, Fujimoto K, Imamura H, Ushiki A, Kanda S, Tsushima K, Kubo H, Yamaya M, Kubo K: L-carbocisteine reduces neutrophil elastase-induced mucin production. Respiratory physiology \& neurobiology 2009; 167: 214216.

(Received, November 18, 2017)

(Accepted, March 6, 2018) 